Proc. XIX International School of Semiconducting Compounds, Jaszowiec 1990

\title{
SUBMILIMETER MAGNETOSPECTROSCOPY ON ZnFeSe
}

\author{
R. Krevet, P. Kempf, M. von Ortenberg \\ Institut für Halbleiterphysik und Optik, Technische Universität Braunschweig, \\ Mendelssohnstr. 3, D-3300 Braunschweig, Germany
}

AND A. TWARDOWSKI

Institute of Experimental Physics, Warsaw University, Hoża 69, 00-681 Warszawa, Poland

(Received August 8, 1990)

\begin{abstract}
We report FIR laser spectroscopy study of $\mathrm{Zn}_{1-x} \mathrm{Fe}_{x}$ Se $(x<0.06)$ Semimagnetic Semiconductor at the temperature range of $2-26 \mathrm{~K}$ and magnetic fields up to $18 \mathrm{~T}$.
\end{abstract}

PACS numbers: 78.30.Fs, 71.70.Ej

One of the central problems of Fe-based Semimagnetic Semiconductor (SMSC) is the information about the energy structure of the $\mathrm{Fe}^{++}$ion ground term $\left({ }^{5} E\right.$, split into a singlet $A_{1}$, a triplet $T_{1}$, a doublet $E$, a triplet $T_{2}$ and a singlet $A_{2}$ [1]). A useful tool to study this structure is far infrared (FIR) spectroscopy $[1,2]$. Recently, an investigation of Fe-type SMSC has been reported [3, 4], however it dealt with rather low $\mathrm{Fe}$ concentrations. In this paper we present complementary results of FIR laser spectroscopy for $\mathrm{Zn}_{1-x} \mathrm{Fe}_{x} S e(x<0.06)$ in the temperature range $2<T<26 \mathrm{~K}$ and magnetic fields up to $18 \mathrm{~T}$.

In Fig. 1 we display resonant energies observed experimentally as a function of magnetic field. The previously observed $[3,4]$ transitions $A_{1}-T_{1}$ and $A_{1}-E$ are clearly visible also for higher $x$, although the spectra are broader than those obtained for low $x$. The experimental energies match well the calculated energy level differences for isolated, i.e. not interacting $\mathrm{Fe}^{++}$ion (Fig. 1). We notice that the line observed at $14.3 \mathrm{~cm}^{-1}$ coincides with the lowest transition $A_{1}-T_{1}$. However, this transition is only weakly dependent on the field and therefore should not be observed in laser spectroscopy as a resonance (fixed energy of laser line). In that respect the origin of $14.3 \mathrm{~cm}^{-1}$ line is still not clear. 


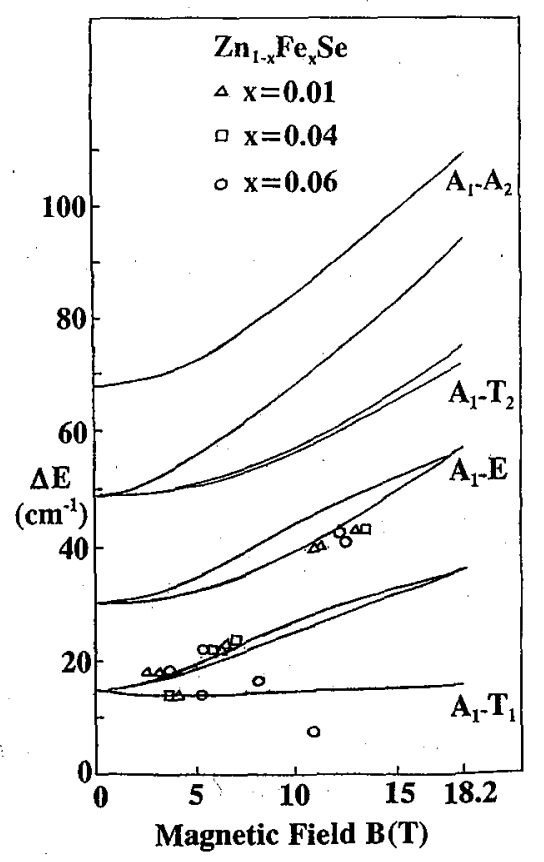

Fig. 1. The experimentally observed resonance energies as a function of magnetic field; the lines present calculated energies of transitions: solid lines - model from [1] with $D q=293 \mathrm{~cm}^{-1}$ and $\lambda=-95 \mathrm{~cm}^{-1}$, dotted lines - model fron [2] with $D q=294 \mathrm{~cm}^{-1}$ and $\lambda=-88 \mathrm{~cm}^{-1}$; parameters were obtained by fitting to the zero-field energies of $A_{1}-T_{1}, A_{1}-T_{2}$ transitions as well as zero phonon line of ${ }^{5} E-{ }^{5} T$ transition.

For $\mathrm{Zn}_{0.94} \mathrm{Fe}_{0.06} \mathrm{Se}$ we observed a weak and broad line at $8.2 \mathrm{~cm}^{-1}$ (Fig. 1). This line disappears at temperatures $T>5 \mathrm{~K}$ and is not visible for lower $\mathrm{Fe}$ concentrations. We stress that the transition energy is roughly two times smaller than the lowest transition energy expected for isolated Fe ion. A possible candidate for the $8.2 \mathrm{~cm}^{-1}$ lines is the transition between the ground and the first excited states of a $\mathrm{Fe}-\mathrm{Fe}$ pair coupled by exchange interaction. In $\mathrm{ZnFeSe}$, calculated energy of this transition is about $8-9 \mathrm{~cm}^{-1}$ [5]. An increasing contribution of pair and larger clusters to FIR absorption should be expected for $x>0.03$ and is indeed exhibited by the changes of FIR absorption spectra shape [6, 3] as well as by the temperature variation of the spectra (Fig. 2). We believe, however, that the available data are too preliminary to establish the nature of $8.2 \mathrm{~cm}^{-1}$ line. 


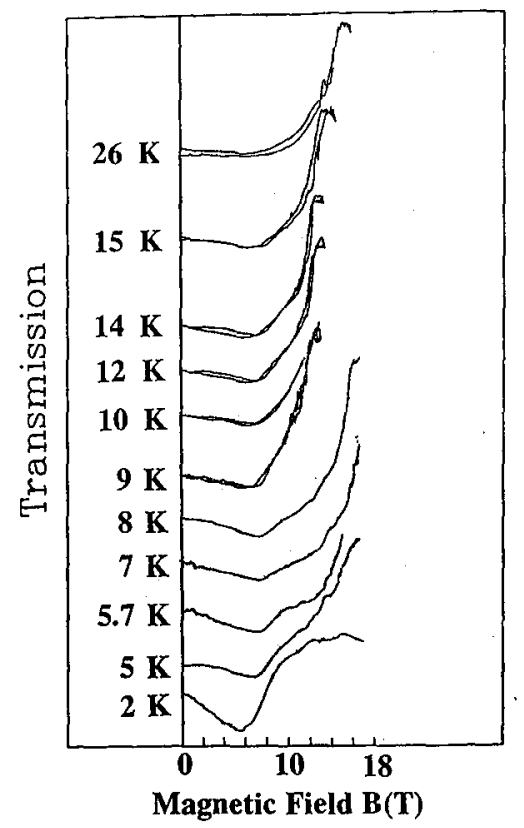

Fig. 2. The experimental FIR transmission spectra of $\mathrm{Zn}_{0.94} \mathrm{Fe}_{0.06} \mathrm{Se}$ as a function of magnetic field for various temperatures. The laser energy was $21.2 \mathrm{~cm}^{-1}$. Curves at $9<T<26 \mathrm{~K}$ show results for upward and downward sweeps of magnetic field.

\section{References}

[1] G.A. Slack, S. Roberts, J.T. Wallin, Phys.Rev. 187, 511 (1969).

[2] J.T. Vallin, G.A. Slack, C.C. Bradley, Phys. Rev. B 2, 4406 (1970).

[3] A.M. Witowski, A. Twardowski, M. Pohlmann, W.J.M. de Jonge, A. Wieck, A. Mycielski, M. Demianiuk, Solid Slate Commun. 70, 27 (1989).

[4] M. Hausenblas, L.M. Claessen, A. Wittlin, A. Twardowski, M. von Ortenberg, W.J.M. de Jonge, P. Wyder, Solid State Commun. 72, 253 (1989).

[5] A. Twardowski, H.J.M. Swagten, T.F.H. v.d. Wetering, W.J.M. de Jonge, Solid State Commun. 65, 235 (1988).

[6] A. Twardowski, H.J.M. Swagten, W.J.M. de Jonge, in Proc. 19th Internat. Conf. on the Physics of Semiconductors, Warszawa 1988, ed. W. Zawadzki, Institute of Physics, Polish Academy of Sciences, Warsaw 1989, p. 1543. 